Aceptado: Mayo 2018

\title{
Condición física, dieta y ocio digital según práctica de actividad física en estudiantes universitarios de Granada
}

\section{Physical fitness, diet and digital leisure depending on physical activity in university students from Granada}

\author{
Ramón Chacón-Cuberos ${ }^{1}$, Félix Zurita-Ortega ${ }^{2}$, José Luis Ubago-Jiménez ${ }^{2}$, Gabriel González-Valero ${ }^{2 *}$ y María Sánchez- \\ Zafra $^{2}$ \\ 1 Departamento de Didácticas Integradas. Area de Expresión Corporal. Universidad de Huelva. Huelva (España). \\ 2 Departamento de Didáctica de la Expresión Musical, Plástica y Corporal. Universidad de Granada. Granada (España).
}

\begin{abstract}
Resumen: El presente estudio pretende analizar la composición corporal, el $\mathrm{VO}_{2}$ max, flexibilidad, dieta y uso problemático de videojuegos según el sexo y la práctica de actividad físico-deportiva en una muestra de estudiantes universitarios de la provincia de Granada. El diseńo del estudio fue descriptivo y de corte transversal. En él participaron un total de 138 sujetos con una edad media de 22,60 $\pm 2,79$ ańos y una distribución por sexo del $65,9 \%(n=91)$ para los hombres y del 34,1\% $(n=47)$ eran mujeres. Como principales resultados se observó que los varones poseían un mayor $\mathrm{VO}_{2}$ max y masa magra, además de mostrar un uso problemático de videojuegos más elevado. Asimismo, los estudiantes universitarios que practican actividad física más de tres horas semanales poseían una mayor masa magra y $\mathrm{VO}_{2}$ max. Del mismo modo, el uso problemático de videojuegos es más elevado en los jóvenes físicamente activos por su perfil tecno-activo.

Palabras clave: Actividad Física; Deporte; Dieta; Videojuegos; Condición Física.
\end{abstract}

Abstract: The present study aims to analyze the body composition, VO2max, flexibility, diet and problematic use of video games according to sex and the practice of physical-sport activity in a sample of university students from the province of Granada. A total of 138 subjects participated in this descriptive and cross-sectional study with a mean age of $22.60 \pm 2.79$ years and a gender distribution of $65.9 \%(\mathrm{n}=91)$ for men and $34.1 \%(\mathrm{n}=$ 47) for women. The main results showed that males had a higher VO2max and lean mass, in addition to showing a higher problematic use of video games. Moreover, university students who practice physical activity more than three hours a week had a higher lean mass and VO2max. Similarly, the problematic use of video games is higher in young people who are physically active because of their techno-active profile.

Keywords: Physical activity; Sport; Diet; Video games; Physical Fitness.

\section{Introducción}

Etapa universitaria, caracterizada como el periodo en el cual los adultos jóvenes adquieren los grados profesionalizantes que les permiten insertarse en el mercado laboral, se aglutina en una fase de gran complejidad para los jóvenes como es la adultez emergente (Chacón et al., 2017). Este periodo se define como la última fase de la adolescencia según Arnett (2014) y si bien ya se han producido todos los cambios biológicos y madurativos propios de esta etapa, aun no se han instalado por completo las característica socio-afectivas y culturales propias de la adultez (Castro, 2016; O'Connor et al., 2011). Por ello, resulta esencial el estudio y promoción de hábitos físico-saludables en esta etapa, ya que ello ayudará no solo a la mejora del estado de salud general, sino en la configuración de hábitos que saludables que se repetirán en la adultez (López, Ahmed, \& Díaz, 2017).

Con el objetivo de lograr tal fin, la práctica de actividad

Dirección para correspondencia [Correspodence address]: Gabriel González-Valero. Departamento de Didáctica de la Expresión Musical, Plástica y Corporal. Universidad de Granada. Granada (España).E-mail: ggvalero@ugr.es físico-deportiva se constituye como el eje central para la mejora de salud. De hecho, múltiples trabajos de investigación han puesto en alza muchos de sus beneficios, entre los cuales destacan la mejora de la composición corporal, la disminución del riesgo de padecer enfermedades cardiovasculares, osteoporosis o diabetes (Arem et al., 2015; Reiner, Niermann, Jekauc y Woll, 2013), así como la mejora de la salud cognitiva y emocional o social (Chacón et al., 2018; Staiano y Calvert, 2011). Para ello, resulta esencial cumplir con los mínimos establecidos de actividad física diaria recomendada por la Organización Mundial de la Salud (OMS, 2018), los cuales se establecen en al menos 150 minutos de actividad física de intensidad moderada a la semana para personas adultas.

De forma muy ligada a la práctica físico-deporte aparece el ocio digital sedentario, mostrando una relación inversa entre ambos factores (Chacón et al., 2017). El uso de videojuegos y otros dispositivos de pantalla se ha expandido en la sociedad actual, acarreando graves consecuencias para la salud (Chaput, LeBlanc, Goldfield y Tremblay, 2013; Foley y Maddison, 2010). Concretamente el uso de estos dispositivos disminuye notoriamente el tiempo invertido en prác- 
tica deportiva, aumentando los niveles de sedentarismo que ocasionan mayores índices de sobrepeso y obesidad (Espejo et al., 2015). Asimismo, se asocian con otros problemas para la salud, como son estados de estrés, pérdida de capacidad de autocontrol, patología ocular, mala alimentación o problemas óseo-articulares (Chacón et al., 2016; Gil, Oberst, Del Valle y Chamarro, 2015).

Junto con la práctica de actividad física, seguir una dieta saludable resulta esencial para la mejora de la salud. En este sentido, la Dieta Mediterránea (DM) se constituye como uno de los modelos dietéticos que mayores beneficios reportan para la salud, los cuales han sido ampliamente demostrados (Chacón et al., 2016; Muros, Cofre-Bolados, Arriscado, Zurita y Knox, 2017). Esta dieta se caracteriza por un consumo elevado de antioxidantes naturales a través de fruta, verdura, legumbres, aceite de oliva y pescado, así como un escaso consumo de carnes y lácteos. Concretamente, varios estudios han demostrado la existencia de una relación positiva entre una elevada adherencia a la DM y el estado de salud y bienestar de las personas (Grao-Cruces, Nuviala, Fernández-Martínez y Martínez-López, 2015; Knox y Muros, 2017).

Por tanto, este estudio persigue como principales objetivos:

- Describir la composición corporal, flexibilidad, $\mathrm{VO}_{2} \max$, dieta y uso problemático de videojuegos en una muestra de estudiantes universitarios según su sexo.

- Analizar las asociaciones dadas entre estas variables y la práctica de actividad física semanal de los estudiantes universitarios.

\section{Material y método}

\section{Diseño y participantes}

El diseño de la investigación fue de tipo descriptivo y corte transversal, con una medición única en un único grupo. La muestra de estudio se confeccionó seleccionando grupos naturales de la titulación universitaria de Grado en Educación Primaria con Mención en Educación Física, considerando su aleatorización por grupos naturales -Cluster-RandomizedControlled Trial- (Merino-Marban, Mayorga-Vega, Fernandez-Rodríguez, Estrada y Viciana, 2015). La muestra estuvo constituida por 138 participantes, de los cuales un 65,9\% eran hombres $(n=91)$ y un $34,1 \%$ eran mujeres $(n=47)$. La edad media fue de 22,60 (DT = 2,79), con una edad mínima de 20 ańos y máxima de 38. La selección de participantes fue realizada por conveniencia, concretando como criterio de inclusión cursar último curso del grado en Educación Primaria con Mención en Educación Física.

\section{Instrumentos}

La composición corporal - porcentaje de masa grasa y masa magra- fue determinada mediante balanza electrónica, utilizando el modelo Tanita $\mathrm{TBF} 300^{\circ}$. Este modelo requiere de las variables sexo, edad y estatura, siendo esta última valorada mediante un estadiómetro Holtain $\mathrm{LTD}^{\oplus}$ y siguiendo el protocolo establecido por Portao, Bescós, Irurtia, Cacciatori y Vallejo (2009).

Se valoró la flexibilidad mediante el test del cajón para la flexión profunda de tronco (Canda, Gómez y Heras, 2004). El protocolo consiste en desplazar un cajón de cartón que está situado en una línea recta trazada paralela e inmediata a los talones del sujeto, sin desplazar los pies y permitiendo la flexión de rodillas. La posición final debe ser mantenida y sostenida, procediendo a la medición de la distancia alcanzada en centímetros.

Adhesión a la dieta Mediterránea, la cual se valoró mediante el cuestionario KIDMED (Serrá-Majem et al., 2004). Este test cuenta con 16 ítems de tipo dicotómico de respuesta afirmativa o negativa (Ej: 1 . Toma una fruta o un zumo natural todos los días), los cuales hacen referencia a patrones asociados el modelo mediterráneo. Cuatro de estos se forma los ítems poseen connotación negativa (-1), mientras que los doce restantes se valoran de forma positiva $(+1)$, oscilando la puntuación final entre $-4 \mathrm{y}+12$. Para este cuestionario se obtuvo una consistencia interna de $\alpha=.773$.

Cuestionario de Experiencias Relacionadas con Videojuegos (CERV), el cual fue validado por Chamarro et al. (2014) en adolescentes. Este test valora el uso problemático de videojuegos y se compone de 17 ítems de connotación negativa (Ej: 1. ¿Hasta qué punto te sientes inquieto por temas relacionados con los videojuegos?), los cuales son puntuados a través de una escala Likert de cuatro opciones ( 1 = Casi Nunca; 2 = Algunas veces; 3 = Bastantes veces; 4 = Casi siempre). $\mathrm{La}$ fiabilidad de este instrumento ha sido de $\alpha=.896$.

El $\mathrm{Vo}_{2}$ máx fue medido de forma indirecta a partir del test "Meter Shuttle Run Test (20mSRT) (Rey, Maïano, Nicol, Mercier y Vallier, 2016). Este test es de carácter incremental máximo y consiste en hacer un recorrido de ida y vuelta con una distancia de 20 metros siguiendo una velocidad marcada por el protocolo $20 \mathrm{mSRT}$. La velocidad inicial marcada por el ritmo es de $8 \mathrm{~km} / \mathrm{h}$, la cual incremente $0,5 \mathrm{~km} / \mathrm{h}$ cada minuto. Para calcular el $\mathrm{VO}_{2}$ máx indirectamente se emplea la velocidad alcanzada en la última etapa (VFA), empleando la siguiente fórmula: $\mathrm{VO}_{2}$ máx $(\mathrm{ml} / \mathrm{min} / \mathrm{kg})=(6 \mathrm{x} \mathrm{FA})-27,4$ (García y Secchi, 2014).

Se utilizó un cuestionario $A d H o c$ para el registro de las variables de tipo sociodemográfico -sexo, edad, etc.-, así como aquellas relacionadas con la práctica de actividad física -se considera la práctica de actividades físico-deportivas por un periodo superior a 3 horas semanales-. 


\section{Procedimiento}

En primer lugar, se procedió a solicitar la colaboración de los participantes a través de una carta informativa elaborada desde el Área de Corporal de la Universidad de Granada. En esta se detallaba la naturaleza y objetivos del estudio a realizar, así como se solicitaba el consentimiento de los participantes.

Posteriormente se realizó la recogida de datos. Un total 151 estudiantes universitarios tomaron parte en el estudio, teniendo que invalidar 13 cuestionarios por encontrarse mal cumplimentados; configurando una muestra final de 138 participantes. La aplicación de los instrumentos se realizó en horario lectivo en el centro descrito sin que ocurriese ningún tipo de incidencia. Asimismo, los investigadores estuvieron presentes con el fin de asegurar una correcta aplicación de los instrumentos.

Se aseguró el anonimato a todos los estudiantes, los cuales participaron de manera voluntaria y respetando el acuerdo sobre ética de Investigación de Helsinki. El Comité Ético de Investigación de la Universidad de Granada aprobó este estudio (462/CEIH/2017).

\section{Análisis de los datos}

El análisis estadístico se ha realizado a través del software IBM SPSS ${ }^{\circledR}$ 22.0. Para los descriptivos básicos se emplearon frecuencias y medias, mientras que para el estudio de relaciones entre variables se ha utilizado prueba $\mathrm{T}$ de muestras independientes. La normalidad de los datos se realizó mediante el test de Kolmogorov-Smirnov, utilizando la corrección de Lillieforts y la homocedasticidad a través del test de Levene. La fiabilidad interna de los instrumentos empleados fue valorada mediante el coeficiente alfa de Cronbach, fijando el Índice de Confiabilidad en el 95,5\%.

\section{Resultados}

La Tabla 1 muestra las puntuaciones medias obtenidas en los diferentes indicadores de salud según el sexo de los participantes. Se obtienen diferencias estadísticamente significativas para la masa magra $(\mathrm{p}<.001)$, el uso problemático de videojuegos $(\mathrm{p}<.001)$ y el $\mathrm{VO}_{2} \max (\mathrm{p}<.001)$. Concretamente, se observa que los varones obtienen puntuaciones medias más elevadas que las mujeres en la masa magra $(62,79$ $\pm 6,56$ vs. 42,49 $\pm 6,39$ ), el uso problemático de videojuegos $(36,37 \pm 11,99$ vs. $25,09 \pm 5,82)$ y el $\mathrm{VO}_{2} \max (54,68 \pm 7,16$ vs. $46,03 \pm 7,22)$.

Tabla 1. Indicadores de salud en función del sexo.

\begin{tabular}{|c|c|c|c|c|c|c|c|}
\hline & & & & & \multicolumn{2}{|c|}{ Prueba de Levene } & Prueba T \\
\hline & Sexo & $\mathrm{N}$ & M & DT & $\mathrm{F}$ & Sig. & Sig. (bilateral) \\
\hline \multirow{2}{*}{ Masa Grasa } & Hombre & 91 & 11,65 & 5,43 & \multirow{2}{*}{0,980} & \multirow{2}{*}{0,324} & \multirow{2}{*}{.467} \\
\hline & Mujer & 47 & 12,36 & 5,51 & & & \\
\hline \multirow{2}{*}{ Masa Magra } & Hombre & 91 & 62,79 & 6,56 & \multirow{2}{*}{5,034} & \multirow{2}{*}{0,026} & \multirow{2}{*}{$.000^{*}$} \\
\hline & Mujer & 47 & 42,49 & 6,39 & & & \\
\hline \multirow{2}{*}{ Flexibilidad } & Hombre & 91 & 28,21 & 7,90 & \multirow{2}{*}{0,277} & \multirow{2}{*}{0,599} & \multirow{2}{*}{.405} \\
\hline & Mujer & 47 & 29,44 & 8,79 & & & \\
\hline \multirow{2}{*}{ KIDMED } & Hombre & 91 & 6,44 & 2,40 & \multirow{2}{*}{0,005} & \multirow{2}{*}{0,941} & \multirow{2}{*}{.687} \\
\hline & Mujer & 47 & 6,62 & 2,46 & & & \\
\hline \multirow{2}{*}{ Videojuegos } & Hombre & 91 & 36,37 & 11,99 & \multirow{2}{*}{25,175} & \multirow{2}{*}{0,000} & \multirow{2}{*}{$.000^{*}$} \\
\hline & Mujer & 47 & 25,09 & 5,82 & & & \\
\hline \multirow{2}{*}{$\mathrm{VO} 2 \max$} & Hombre & 91 & 54,68 & 7,16 & \multirow{2}{*}{0,000} & \multirow{2}{*}{0,987} & \multirow{2}{*}{$.000^{*}$} \\
\hline & Mujer & 47 & 46,03 & 7,22 & & & \\
\hline
\end{tabular}

La Tabla 2 muestra las puntuaciones medias obtenidas en los diferentes indicadores de salud según la práctica de actividad física que realizan los participantes. Se obtienen diferencias estadísticamente significativas para la masa magra $(\mathrm{p}=.039)$, el uso problemático de videojuegos $(\mathrm{p}=.030)$ y el $\mathrm{VO}_{2} \max (\mathrm{p}$ $<.001)$. Concretamente, se observa que los sujetos que practi- can actividad física o deporte al menos tres horas semanales obtienen puntuaciones medias más elevadas que los que no lo hacen en la masa magra $(56,66 \pm 11,54$ vs. 50,62 $\pm 11,07)$, el uso problemático de videojuegos $(33,36 \pm 11,88$ vs. $27,00 \pm$ $7,73)$ y el $\mathrm{VO}_{2} \max (52,82 \pm 7,71$ vs. $44,51 \pm 8,31)$. 
Tabla 2. Indicadores de salud en función de la práctica de actividad física.

\begin{tabular}{|c|c|c|c|c|c|c|c|}
\hline & & & & & \multicolumn{2}{|c|}{ Prueba de Levene } & Prueba T \\
\hline & $\mathrm{AF}$ & $\mathrm{N}$ & M & DT & $\mathrm{F}$ & Sig. & Sig. (bilateral) \\
\hline \multirow{2}{*}{ Masa Grasa } & $\mathrm{Si}$ & 120 & 11,80 & 5,18 & \multirow{2}{*}{3,568} & \multirow{2}{*}{.610} & \multirow{2}{*}{.613} \\
\hline & No & 18 & 12,50 & 7,15 & & & \\
\hline \multirow{2}{*}{ Masa Magra } & $\mathrm{Si}$ & 120 & 56,66 & 11,54 & \multirow{2}{*}{0,002} & \multirow{2}{*}{.965} & \multirow{2}{*}{$.039^{*}$} \\
\hline & No & 18 & 50,62 & 11,07 & & & \\
\hline \multirow{2}{*}{ Flexibilidad } & $\mathrm{Si}$ & 120 & 28,73 & 8,42 & \multirow{2}{*}{0,816} & \multirow{2}{*}{.368} & \multirow{2}{*}{.706} \\
\hline & No & 18 & 27,94 & 6,76 & & & \\
\hline \multirow{2}{*}{ KIDMED } & $\mathrm{Si}$ & 120 & 6,58 & 2,26 & \multirow{2}{*}{1,870} & \multirow{2}{*}{.940} & \multirow{2}{*}{.349} \\
\hline & No & 18 & 6,00 & 3,32 & & & \\
\hline \multirow{2}{*}{ Videojuegos } & $\mathrm{Si}$ & 120 & 33,36 & 11,88 & \multirow{2}{*}{3,735} & \multirow{2}{*}{.055} & \multirow{2}{*}{$.030^{*}$} \\
\hline & No & 18 & 27,00 & 7,73 & & & \\
\hline \multirow{2}{*}{$\mathrm{VO} 2 \max$} & $\mathrm{Si}$ & 120 & 52,82 & 7,71 & \multirow{2}{*}{0,193} & \multirow{2}{*}{.661} & \multirow{2}{*}{$.000^{*}$} \\
\hline & No & 18 & 44,51 & 8,31 & & & \\
\hline
\end{tabular}

\section{Discusión}

El presente estudio persiguió como principales objetivos analizar las diferencias dadas en la masa grasa y magra, flexibilidad, consumo máximo de oxígeno, calidad de la dieta seguida y uso problemático de videojuegos en una muestra de estudiantes universitarios de la provincia de Granada, así como las asociaciones dadas entre estas variables y la práctica de actividad físico-deportiva. En esta línea, algunos estudios de similares características son los realizados por Chacón-Cuberos, Puertas-Molero y Pérez-Cortés (2017), Cutillas, Herrero, San Eustaquio, Zamora y Pérez-Llamas (2013), Lepp, Barkley, Sanders, Rebold y Gates (2013) y Sacheck, Kuder y Economos (2010).

Se observó que los hombres poseían una mayor puntuación media de masa magra con respecto a las mujeres, mientras que estas poseía un valor ligeramente superior en masa grasa, aunque sin ser estadísticamente significativo. Estos resultados están en consonancia con lo establecido por Geek y Shen (2009) y Goodpaster et al. (2006), quienes estudian las diferencias antropométricas y de la composición corporal en función del sexo sujetos de diferente edad. Concretamente, la justificación estos resultados poder deberse a las diferencias hormonales según el sexo, dado que los varones poseen una mayor concentración de hormonas androgénicas como la testosterona, la cual favorece el desarrollo de la masa muscular (Smith, Sommer, Starkoff y Devor, 2013).

En relación a la flexibilidad de los sujetos no se obtuvieron diferencias estadísticamente significativas según el sexo, aunque puede apreciarse una cierta tendencia que muestra que las mujeres poseen una mayor capacidad de flexión de tronco. Geer y Shen (2009) lo justifican a través de la mayor cantidad de grasa corporal que poseen las mujeres asociada a factores hormonales, lo cual confiere una mayor maleabilidad a los tejidos blandos que configuran las articulaciones de las mismas permitiéndoles alcanzar un mayor rango de movilidad articular (Moen, Kelly, Tranby y Huang, 2011). En cambio, el $\mathrm{VO}_{2}$ max si reveló diferencias estadísticas a través de valores más elevados para los hombres, lo que se debe a su mayor volumen sistólico, capacidad pulmonar, masa magra, etc. (Lepp et al., 2013; Reiner et al., 2013).

No se observaron diferencias estadísticas en relación a la calidad dieta, aunque nuevamente las mujeres presentaban valores más elevados; lo cual puede explicarse por la mayor relevancia que da este sexo al cuidado de la imagen corporal a través de la alimentación (Cutillas et al., 2013; Kantanista, Osiński, Borowiec, Tomczak y Król-Zielińska, 2015; Knox y Muros, 2017). En relación al ocio de pantalla, se concretó que los varones realizaban un uso problemático más elevado, ya que este sexo posee una mayor capacidad de acción hacia estos dispositivos por las necesidades de competencia y los sistemas de recompensa que crean (Chacón et al., 2017; Espejo et al., 2015).

Analizando los indicadores de salud mencionados según la práctica de actividad física pudo observarse que aquellos jóvenes que realizan más de tres horas semanales de actividad físico-deportiva eran los que poseían un mayor $\mathrm{VO}_{2}$ max. Este término hace referencia a la cantidad de oxígeno que nuestro organismo es capaz de transportar en un intervalo de tiempo, por lo que esta asociación puede venir justificada por los mayores niveles de hemoglobina y densidad mitocondrial que produce la práctica deportiva, así como la mejora del gasto cardiaco, la difusión pulmonar o la capilarización de la musculatura (Miranda y Navío, 2013; Moral, 2018; Reiner et al., 2013). Igualmente, se observó que los jóvenes físicamente activos poseían una mayor masa magra, fundamentado en 
las adaptaciones producidas por la práctica de actividad física, destacando la renovación y desarrollo de fibras contráctiles y el aumento de la sección transversal (Reiner et al., 2013; Sacheck et al. 2010).

Para concluir, se observó que los que más actividad física practicaban eran los que poseían un nivel de uso de videojuegos más elevado. Esta perspectiva ya ha sido estudiada por Beltrán, Valencia y Molina (2011) y Chacón et al., 2017, justificándose por el perfil tecno-activo de los jóvenes del siglo XXI. Asimismo, tanto el uso de videojuegos como la práctica deportiva competitiva se han relacionado con muchas de las necesidades de competencia de la teoría de la autodeterminación, configurando motivaciones extrínsecas que favorecen ambas prácticas (Chacón et al., 2017; Foley y Maddison, 2010).

Como principales limitaciones de este trabajo de investigación pueden destacarse las propias de un estudio de tipo descriptivo y corte transversal, ya que este no permite concretar relaciones causa-efecto; aunque resulta eficaz para conocer el estado de una cuestión concreta. El mismo modo cabe destacar la muestra utilizada, la cual no es representativa para la provincia de Granada. Asimismo, se utiliza un muestro por conveniencia, aunque se fundamenta en la aleatorización dada por grupos naturales. Como perspectivas futuras se considera de relevante interés la ampliación de la muestra de estudio con el fin de otorgar mayor fiabilidad a los resultados obtenidos, así como incluir otras variables que permitan definir mejor la influencia de la actividad física en indicadores de salud.

Las conclusiones que presenta este estudio son:

- Los varones poseen un mayor $\mathrm{VO}_{2} \max$ y una mayor cantidad de masa magra. El uso problemático de videojuegos es más común en los hombres.

- Los estudiantes universitarios que practican actividad física más de tres horas semanales poseen una mayor masa magra y $\mathrm{VO}_{2}$ max. Del mismo modo, el uso problemático de videojuegos es más elevado en los jóvenes físicamente activos.

\section{Referencias bibliográficas}

1. Arem, H., Moore, S. C., Patel, A., Hartge, P., De Gonzalez, A. B., Visvanathan, K., ... y Linet, M. S. (2015). Leisure time physical activity and mortality: a detailed pooled analysis of the dose-response relationship. JAMA Internal Medicine, 175(6), 959-967. Doi: 10.1001/jamainternmed.2015.0533.

2. Arnett, J. J. (2014). Adolescence and emerging adulthood. A Cultural Aproach. Fifth Edition. Boston, MA: Pearson.

3. Beltrán, V. J., Valencia, A. y Molina, J. P. (2011). Los videojuegos activos y la salud de los jóvenes: revisión de la investigación. Revista Internacional de Medicina y Ciencias de la Actividad Física y Deporte, 10(41), 203-219.

4. Canda, A. S., Gómez, A. y Heras, E. (2004). Valoración de la flexibilidad de tronco mediante el test del cajón en diferentes modalidades deportivas. Revista Española de Medicina de la Educación Física y el Deporte, 13(4), 148-154.

5. Castro, M. (2016). Análisis de los parámetros psicosociales, conductuales, físico-deportivos y laborales de los adolescentes de Granada. Tesis Doctoral: Universidad de Granada.

6. Castro, R., Pérez, V., Cachón, J. y Zagalaz, M. L. (2016). Valoración de la relación entre Rendimiento Académico y Condición Física en escolares zaragozanos. SPORT TK-Revista EuroAmericana de Ciencias del Deporte, 5(1), 47-53.

7. Chacón, R., Castro, M., Muros, J. J., Espejo, T., Zurita, F. y Linares, M. (2016). Adhesión a la dieta mediterránea en estudiantes universitarios y su relación con los hábitos de ocio digital. Nutrición Hospitalaria, 33(2), 405-410.

8. Chacón, R., Chacón, F., Zurita, F., Cachón, J., Zagalaz, M. L. y Chinchilla, J. J. (2018). Characterization of motivation and type of physicalsport practice in adults through COMPASS profiles. Journal of Human Sport and Exercise, 13(1), 161-173. Doi: 10.14198/jhse.2018.131.16.

9. Chacón, R., Zurita, F., Castro, M., Espejo, T., Martínez, A. y Pérez, A. J. (2017). Motivational climate in sport and its relationship with digital sedentary leisure habits in university students. Saúde e Sociedade, 26(1), 29-39. Doi: 10.1590/S0104-1290201716656.

10. Chacón-Cuberos, R., Puertas-Molero, P., \& Pérez-Cortés, A. J. (2017). Niveles de resiliencia según práctica de actividad física en estudi- antes universitarios de Educación Física. ESHPA - Education, Sport, Health and Physical Activity, 1(1), 59-67. Doi: http://hdl.handle. net/10481/48963.

11. Chamarro, A., Carbonell, X., Manresa, J. M., Muñoz-Mirallles, R., Ortega-González, R., López-Morrón, M. R., Batalla-Martínez, C. y Torán-Monserrat, P. (2014). El Cuestionario de Experiencias Relacionadas con los Videojuegos (CERV): Un instrumento para detectar el uso problemático de videojuegos en adolescentes españoles. Adicciones, 26(4), 303-311. Doi: 10.20882/adicciones.31.

12. Chaput, J. P., LeBlanc, A. G., Goldfield, G. S. y Tremblay, M. S. (2013). Are active video games useful in increasing physical activity and addressing obesity in children? JAMA Pediatrics, 167(7), 677-678. Doi: 10.1001/jamapediatrics.2013.2424.

13. Cutillas, A. B., Herrero, E., San Eustaquio, A. D., Zamora, S. y PérezLlamas, F. (2013). Prevalencia de peso insuficiente, sobrepeso y obesidad, ingesta de energía y perfil calórico de la dieta de estudiantes universitarios de la Comunidad Autónoma de la Región de Murcia (España). Nutrición Hospitalaria, 28(3), 683-689.

14. Espejo, T., Chacón, R., Castro, M., Martínez, A., Zurita, F. y Pinel, C. (2015). Análisis descriptivo del uso problemático y hábitos de consumo de los videojuegos con relación al género en estudiantes universitarios. RELATEC, 14(3), 85-93.

15. Foley, L. y Maddison, R. (2010). Use of active video games to increase physical activity in children: a (virtual) reality? PediatricExerciseScience, 22(1), 7-20. Doi: 10.1123/pes.22.1.7.

16. García, G. C. y Secchi, J. D. (2014). Test corusenavette de 20 metros con etapas de un minuto. Una idea original que perdura hace 30 años.Apunts. Medicina de L'esport, 49(183), 93-103. Doi: 10.1016/j. apunts.2014.06.001.

17. Geer, E. B. y Shen, W. (2009). Gender differences in insulin resistance, body composition, and energy balance. Gender Medicine, 6, 60-75. Doi: 10.1016/j.genm.2009.02.002.

18. Gil, F., Oberst, U., Del Valle, G. y Chamarro, A. (2015). Nuevas tecnologías- ¿Nuevas patologías? El smartphone y el fearofmissingout. Aloma: Revista de Psicologia, Ciències de l'Educació i de l'Esport, 33(2), 73-83. 
19. Goodpaster, B. H., Park, S. W., Harris, T. B., Kritchevsky, S. B., Nevitt, M., Schwartz, A. V., ... y Newman, A. B. (2006). The loss of skeletal muscle strength, mass, and quality in older adults: the health, aging and body composition study. The Journals of Gerontology Series A: Biological Sciences and Medical Sciences, 61(10), 1059-1064. Doi: 10.1093/ gerona/61.10.1059.

20. Grao-Cruces, A., Nuviala, A., Fernández-Martínez, A. y MartínezLópez, E. J. (2015). Relationship of physical activity and sedentarism with tobacco and alcohol consumption, and Mediterranean diet in Spanish teenagers. Nutrición Hospitalaria, 31(4), 1693-1700. Doi: 10.3305/nh.2015.31.4.8256.

21. Kantanista, A., Osiński, W., Borowiec, J., Tomczak, M. y Król Zielińska, M. (2015). Body image, BMI, and physical activity in girls and boys aged 14-16 years. Body Image, 15, 40-43. Doi: 10.1016/j. bodyim.2015.05.001.

22. Knox, E. y Muros, J. J. (2017). Association of lifestyle behaviours with self-esteem through health-related quality of life in Spanish adolescents. European Journal of Pediatrics, 176(5), 621-628. Doi: 10.1007/s00431017-2886.011.00162.x.

23. Lepp, A., Barkley, J. E., Sanders, G. J., Rebold, M. y Gates, P. (2013). The relationship between cell phone use, physical and sedentary activity, and cardiorespiratory fitness in a sample of US college students. International Journal of Behavioral Nutrition and Physical Activity, 10(1), 79. Doi: 10.1186/1479-5868-10-79.

24. López Sánchez, G. F., Ahmed, D., \& Díaz Suárez, A. (2017). Level of habitual physical activity among 13-year-old adolescents from Spain and India. A cross-cultural study. SPORT TK: Revista EuroAmericana de Ciencias del Deporte, 6(1), 67-74.

25. Merino-Marban, R., Mayorga-Vega, D., Fernandez-Rodríguez, E., Estrada, F. y Viciana, J. (2015). Effect of a physical education-based stretching programme on sit-andreach score and its posterior reduction in elementary schoolchildren. European Physical Education Review, 21, 83-92. Doi: 10.1177/1356336X14550942.

26. Miranda, M. D. y Navío, C. (2013). Benefits of exercise for pregnant women. Journal of Sport and Health Research, 5(2), 229-232.

27. Moen, P., Kelly, E. L., Tranby, E. y Huang, Q. (2011). Changing work, changing health: can real work-time flexibility promote health behaviors and well-being?.JournalofHealth and Social Behavior, 52(4), 404429. Doi: 10.1177/0022146511418979.

28. Moral, L. (2018). Nivel de actividad física cardiosaludable en Educación Física en educación primaria: expectativas y algunas evidencias. Sportis.
Scientific Journal of School Sport, Physical Education and Psychomotricity, 4(1), 95-110. Doi: 10.17979/sportis.2018.4.1.2017.

29. Muros, J. J., Cofre-Bolados, C., Arriscado, D., Zurita, F. y Knox, E. (2017). Mediterranean diet adherence is associated with lifestyle, physical fitness, and mental wellness among 10-y-olds in Chile. Nutrition, 35, 87-92. Doi: 10.1016/j.nut.2016.11.002.

30. O’Connor, M., Sanson, A., Hawkins, M. T., Letcher, P., Toumbourou, J. W., Smart, D., ... y Olsson, C. A. (2011). Predictors of positive development in emerging adulthood. Journal of Youth and Adolescence, 40(7), 860-874. Doi: 10.1007/s10964-010-9593-7.

31. OMS (2018). Recomendaciones mundiales sobre la actividad física para la salud. Consultado en la World Wide Web el 06 de febrero de 2018: http://www.who.int/dietphysicalactivity/factsheet_recommendations/ es/

32. Portao, J., Bescós, R., Irurtia, A., Cacciatori, E. yVallejo, L. (2009). Valoración de la grasa corporal en jóvenes físicamente activos: antropometría vs bioimpedancia. Nutrición Hospitalaria, 24(5), 529-534.

33. Reiner, M., Niermann, C., Jekauc, D. y Woll, A. (2013). Long-term health benefits of physical activity-a systematic review of longitudinal studies. BMC Public Health, 13(1), 813-822. Doi: 10.1186/1471-245813-813.

34. Rey, O., Maïano, C., Nicol, C., Mercier, C. S. y Vallier, J. M. (2016) Psycho-Physiological Responses of Obese Adolescents to an Intermittent Run Test Compared with a 20-M Shuttle Run. Journal of Sports Science \& Medicine, 15(3), 451.

35. Sacheck, J. M., Kuder, J. F. y Economos, C. D. (2010). Physical fitness, adiposity, and metabolic risk factors in young college students. Medicine and Science in Sports and Exercise, 42(6), 1039-1044. Doi: 10.1249/ MSS.0b013e3181c9216b.

36. Serrá-Majem, L., Ribas, L., Ngo, J., Ortega, R. M., García, A. PérezRodrigo, C. y Aranceta, J. (2004). Food, youth and the Mediterranean diet in Spain. Development of KIDMED, Mediterranean diet quality index in children and adolescents. Public Health Nutrition, 7(7), 931935. Doi: 10.1079/PHN2004556.

37. Smith, M. M., Sommer, A. J., Starkoff, B. E. y Devor, S. T. (2013). Crossfit-Based High-Intensity Power Training Improves Maximal Aerobic Fitness and Body Composition. The Journal of Strength \& Conditioning Research, 27(11), 3159-3172. Doi: 10.1519/JSC.0b013e318289e59f.

38. Staiano, A. E. y Calvert, S. L. (2011). Exergames for physical education courses: Physical, social, and cognitive benefits. Child DevelopmentPerspectives, 5(2), 93-98. Doi: 10.1111/j.1750-8606.2. 